\title{
Initial Visual Acuity Is an Important Prognostic Factor in Patients with Branch Retinal Vein Occlusion
}

\author{
Jiri Rehak ${ }^{\mathrm{a}}$ Ladislav Dusek $^{\mathrm{b}} \quad$ Oldrich Chrapek $^{\mathrm{a}}$ Evzen Fric $^{\mathrm{a}}$ Matus Rehak $^{\mathrm{c}}$ \\ a Department of Ophthalmology, University Hospital, Palacky University, Olomouc, and ${ }^{\mathrm{b}}$ Institute of Biostatistics \\ and Analyses, Masaryk University, Brno, Czech Republic; ' ${ }^{\circ}$ epartment of Ophthalmology, University of Leipzig, \\ Leipzig, Germany
}

\section{Key Words}

Branch retinal vein occlusion, prognosis - Visual acuity

\begin{abstract}
Purpose: To evaluate the role of initial visual acuity (VA) as a potential prognostic factor for final VA in patients with branch retinal vein occlusion (BRVO). Methods: A retrospective data analysis involving 163 patients with macular edema secondary to BRVO treated according to the recommendations of the Branch Vein Occlusion Study Group was performed using univariate and multivariate logistic regression models, and receiver-operating characteristics analysis. The analyses take factors into account that can potentially influence final visual result: sex, age, type of occlusion (major temporal or macular), grid photocoagulation and ischemia. Results: The final VA $\leq 0.1$ was statistically significantly related to initial VA $\leq 0.16$ and age $>70$ years. Sex, type of occlusion, grid photocoagulation and ischemia did not significantly influence the prediction of final VA based on age and initial VA. Conclusions: The analysis shows that initial VA and age $>70$ years significantly influence the prognosis for final visual results in patients with BRVO.
\end{abstract}

Copyright $\odot 2010$ S. Karger AG, Base
(C) 2010 S. Karger AG, Base

$0030-3747 / 11 / 0454-0204 \$ 38.00 / 0$

Fax +41613061234

E-Mail karger@karger.ch

www.karger.com

\section{Introduction}

In general, branch retinal vein occlusion (BRVO) has a good prognosis; $50-60 \%$ of eyes have been reported to have a final visual acuity (VA) of $\geq 0.5$ even without any treatment [1-3]. Chronic macular edema (ME) and bleeding into the vitreous from neovascularizations account most frequently for a poor final VA [4, 5]. Gutman [5] found that in the natural course of BRVO, only $14 \%$ of eyes with chronic ME retained a VA of $\geq 0.5$, while $86 \%$ had a final VA of $\leq 0.4$. He concluded that chronic ME has a poor prognosis in terms of final VA. A worse visual prognosis has been reported in cases with ischemic $\mathrm{ME}$ than perfused ME in some studies [6,7] but not in others [8]. Generally, it is difficult to predict the visual prognosis for patients with BRVO in the acute phase of the disease. The conflicting reports and small patient numbers in currently published studies make any definitive conclusions about prognostic factors for VA in BRVO patients difficult. Various prognostic factors for BRVO patients have been assessed, e.g. the correlation between macular thickness measured by optical coherence tomography and VA [9] or that between VA and the integrity of the outer photoreceptor layer detected by optical coherence tomography [10]. In patients with central retinal vein occlusion, the initial VA has been shown as a strong prognostic factor for the final VA $[11,12]$. 
In our previously published review [13], we analyzed studies that reported the visual results in patients with BRVO $[1,2,14-16]$. Our review showed that eyes with an initial VA $\geq 0.4$ have a good visual prognosis even without any treatment. The percentage of final VA $\leq 0.1$ was significantly higher $(30-83 \%)$ in patients with an initial VA $\leq 0.1$ than the $0-25 \%$ of eyes in those with an initial VA $\geq 0.4$ [13]. However, methodological differences in the studies analyzed obfuscate definitive conclusions.

For this reason, we realized a retrospective study on our own BRVO patient group with the aim to analyze the role of pretreatment VA as a potential prognostic factor for final VA. Further, we evaluated whether patient sex, age, type of occlusion (major temporal or macular), ischemic status or use of grid laser photocoagulation significantly contribute to the prediction of final VA in BRVO.

\section{Material and Methods}

The retrospective data analysis investigated the relation between initial and final VA in patients with BRVO with a follow-up of 1 year. In total, 221 eyes of 221 patients were assigned to the treatment schedule according to the recommendations of the Branch Vein Occlusion Study Group (BVO SG) in the period from 1987 to 2007 at the Department of Ophthalmology, Palacky University, Olomouc. All these patients were screened for eligibility for analysis in the present study. Additional to ophthalmic laser treatment, all cases also had subsidiary medical therapy with 100 mg pentoxifylline $(3 \times 1), 100 \mathrm{mg}$ salicylic acid $(1 \times 1)$ and ascorbic acid $(3 \times 1)$. Eyes with a BRVO of less than 1 month duration, and $\mathrm{ME}$ confirmed on stereoscopic fundus examination and fluorescein angiography (macular leakage) were included in the analysis. In patients screened after June 2002, ME was also confirmed using optical coherence tomography. Prior to inclusion in the study, all patients underwent a complete ophthalmological examination including the best-corrected VA determined on Snellen charts, slitlamp examination, applanation tonometry and fundus examination in dilated pupils. These examinations were repeated at 3-, 6- and 12-month visits. Fluorescein angiography and fundus photodocumentation were performed at the baseline visit and at 6- and 12-month visits. Grid laser photocoagulation was performed in patients with a VA of $\leq 0.5$ of at least 3 months' duration after inclusion into the study if $\mathrm{ME}$ and/or sufficient absorption of macular hemorrhage was present. Argon green laser was used, $100-\mu \mathrm{m}$ spots for 100-200 ms and 0.5-1 burn width apart.

Exclusion criteria for evaluation in the study were: media opacities, previous laser or surgical treatment, or any other ocular disease involving visual function and incomplete visit schedule. Patients who underwent any other treatment of ME (e.g. vitrectomy, arteriolar crimping technique, intravitreal application of steroids or anti-vascular-endothelial-growth-factor drugs) were also excluded from the statistical analysis.

The primary investigated endpoint was the final VA defined as VA 12 months after the baseline visit. The prevalence of a final VA $\leq 0.1$ was evaluated separately. Further, the study took factors into account which can potentially influence final VA such as patient age, sex, localization of occlusion (major temporal or macular BRVO), use of grid photocoagulation and presence of retinal ischemia. Major BRVO was defined as BRVO of the temporal vein branch. If the clinical signs of the occlusion were limited to the macular region and 1 or more of the macular venules were occluded, the patient was considered as having 'macular BRVO'. Ischemic BRVO was defined according to the classification of the BVO SG [4] as occlusion with capillary nonperfusion larger than 5 disk diameters determined on fluorescein angiography. In the case of large intraretinal hemorrhage preventing evaluation of the ischemic status, the patient was classified as showing ischemic BRVO if cotton wool spots were present.

\section{Statistics}

Standard measures of summary statistics were used to describe data: relative and absolute frequencies, median and 5th-95th percentile range. Robust nonparametric Kruskal-Wallis and MannWhitney $U$ tests were used to evaluate the differences among groups of patients in VA and age. The ML $\chi^{2}$ and Fisher exact tests were used to test the differences in categorical parameters (gender, categories of VA, occurrence of ischemia, type of BRVO major/ macular and grid photocoagulation yes/no). The diagnostic predictive power of all examined parameters was finally assessed on the basis of the receiver-operating characteristic (ROC) curves using categories of VA in 1 year as endpoint. The ROC analysis was performed using an ROC web calculator [17] for curve fitting, SPSS 17.02 [18] for the area-under-the-curve computation and testing and Medcalc 11.1.0.0 (Medcalc Software 1993-2009) for computation of confidence intervals for sensitivity and specificity. The computation was based on binormal distribution assumptions. Both univariate and multivariate logistic regression strategies were applied to quantify the association of examined prognostic factors with VA in 1 year. The odds ratio with $95 \%$ confidence intervals was estimated and tested in the Wald $\chi^{2}$ test. Parameters with potential risk power (providing at least $\mathrm{p}<0.10$ in univariate logistic regression) were then examined for mutual correlation, and interaction terms were coded and tested for significantly correlated pairs of variables. Effective cutoff values of continuous variables (age, VA) were optimized on the basis of the ROC analysis. The final set of potential prognostic factors and interaction terms (coded as binary variables according to the cutoff points) were subjected to a stepwise selection algorithm in multivariate logistic regression (driven by the maximum likelihood ratio test).

\section{Results}

The total sample comprised 221 eyes. Forty-seven patients were excluded from the statistical analysis according to the defined exclusion criteria. A further 11 patients were excluded due to loss to follow-up for at least 12 months.

A total of 163 eyes of 163 patients ( 84 female and 79 male) were analyzed. The mean age of all patients was 62 years (range $47-80$ years). The baseline characteristics of all investigated patients are shown in table 1 . The changes in best-corrected VA during the follow-up of 1 year in 


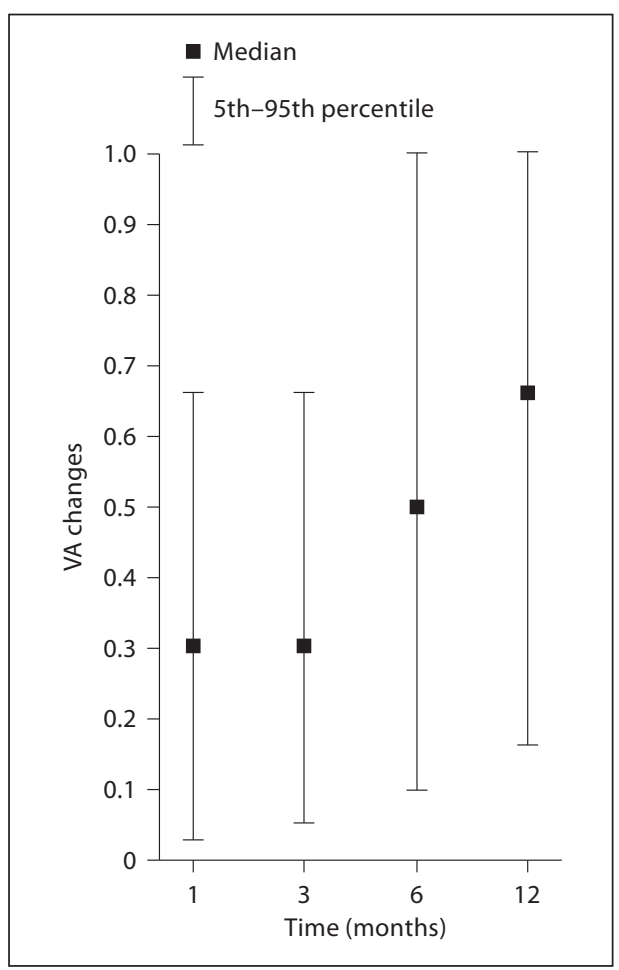

Fig. 1. Summary of the changes of VA in all analyzed patients.

all analyzed patients are shown in figure 1 . The median value of initial VA in patients without laser treatment was 0.42 (5th-95th percentile range: $0.016-1.0$ ) and the median value of final VA after 1 year was $0.61(0.016-1.0)$. Treatment with grid laser photocoagulation was performed in 71 eyes (43.6\%). The median value of initial VA in these patients was $0.27(0.016-0.5)$ and the median value of final VA 1 year after inclusion into the study was 0.43 (0.016-1.0). In 35 eyes (21.5\%), repeated grid laser photocoagulation was done. The grid photocoagulation was repeated in 21 eyes (12.9\%) once, in 10 eyes (6.1\%) twice and in 4 eyes (2.5\%) 3 times. Ninety-two eyes (56.4\%) were observed only and did not receive grid photocoagulation because the BVO SG criteria for grid laser treatment were not met. Scatter laser photocoagulation in areas of retinal nonperfusion was required due to the presence of neovascularization of the disk or elsewhere in $28(17.2 \%)$ of all eyes: in $8(28.6 \%)$ eyes treated with grid photocoagulation and in $20(71.4 \%)$ observed eyes. In 3 $(1.8 \%)$ cases, we noted temporary intravitreal hemorrhage ( 1 eye from the grid laser group and 2 eyes from the observed group). VA $\leq 0.1$ at the 1 -year visit $(\mathrm{n}=37)$ was predominantly associated with dry macula and severe changes in the retinal pigment epithelial layer (very like-
Table 1. Baseline characteristics of patients

\begin{tabular}{|c|c|c|}
\hline \multirow{2}{*}{$\begin{array}{l}\text { Parameters } \\
\text { Male sex, \% }\end{array}$} & \multicolumn{2}{|c|}{ All patients $(\mathrm{n}=163)$} \\
\hline & 48.5 & $(\mathrm{n}=79)$ \\
\hline Age, years & 62 & (range $47-80$ ) \\
\hline$>70$ years, $\%$ & 23.3 & $(\mathrm{n}=38)$ \\
\hline Ischemia, \% & 34.4 & $(n=56)$ \\
\hline \multicolumn{3}{|l|}{ Patients underwent grid } \\
\hline laser photocoagulation, $\%$ & 43.6 & $(\mathrm{n}=71)$ \\
\hline Major BRVO, \% & 80.4 & $(\mathrm{n}=131)$ \\
\hline Initial VA & 0.300 & (range $0.016-1.00$ ) \\
\hline$\leq 0.1, \%$ & 27.6 & $(n=45)$ \\
\hline Final VA in 1 year & 0.66 & (range $0.030-1.00$ ) \\
\hline$\leq 0.1, \%$ & 22.7 & $(\mathrm{n}=37)$ \\
\hline
\end{tabular}

Categorical variables are summarized as number and percentage of a given category; quantitative variables are described by medians and by 5 th-95th percentile ranges.

Table 2. Analysis of risk factors for final VA $\leq 0.1$ based on results of univariate logistic regression models

\begin{tabular}{lcc}
\hline Parameters & \multicolumn{2}{l}{ Final VA in 1 year $\leq 0.10$} \\
\cline { 2 - 3 } & OR & $\mathrm{p}$ \\
\hline Male sex & $0.49(0.23-1.06)$ & 0.067 \\
Age & $1.12(1.07-1.17)$ & $<0.001^{*}$ \\
$\quad>70$ years & $8.42(3.66-19.36)$ & $<0.001^{*}$ \\
Major BRVO & $3.39(0.96-11.95)$ & 0.056 \\
Ischemia & $1.89(0.89-4.03)$ & 0.095 \\
Grid laser & $1.99(0.94-4.22)$ & 0.068 \\
Initial VA $\leq 0.1$ & $9.23(4.03-21.12)$ & $<0.001^{*}$ \\
Initial VA $\leq 0.16$ & $15.17(5.75-40.00)$ & $<0.001^{*}$ \\
\hline
\end{tabular}

$\mathrm{OR}=$ Odds ratio; $95 \%$ confidence intervals are given in parentheses. Statistically significant results $(p<0.05)$ are marked with an asterisk.

ly associated with photoreceptor loss) in 25 cases (67.6\%), and further in 8 patients with persistent chronic ME unimproved after repeated laser treatment (21.6\%) and in 4 cases with insufficient resorption of retinal hemorrhage in the foveal region (10.8\%).

The descriptive analysis showed that although the median value for final VA was $0.66,22.7 \%$ of patients reached a final VA of only $\leq 0.1$ (table 1). Therefore, the risk of this poor final visual outcome is frequent, justifying the development of prognostic models. Initial descriptive analysis also indicated frequent intercorrelations between vari- 
Table 3. Evaluation of diagnostic power of potential prognostic factors in ROC analysis

\begin{tabular}{|c|c|c|c|c|}
\hline \multirow[t]{2}{*}{ Parameters } & \multicolumn{4}{|c|}{ VA in 1 year $\leq 0.10$ as endpoint } \\
\hline & AUC & $\mathrm{p}$ & sensitivity, $\%$ & specificity, $\%$ \\
\hline Male sex & $0.586(0.483-0.689)$ & 0.111 & 64.9 & 52.4 \\
\hline Age $>70$ years & $0.716(0.613-0.820)$ & $<0.001$ & 56.8 & 86.5 \\
\hline Major BRVO & $0.575(0.476-0.673)$ & 0.169 & 91.9 & 23.0 \\
\hline Ischemia & $0.575(0.468-0.682)$ & 0.166 & 45.9 & 69.0 \\
\hline Grid laser photocoagulation & $0.585(0.480-0.690)$ & 0.115 & 56.8 & 60.3 \\
\hline Initial VA $\leq 0.10$ & $0.741(0.642-0.840)$ & $<0.001$ & 64.9 & 74.3 \\
\hline Initial VA $\leq 0.16$ & $0.792(0.710-0.874)$ & $<0.001$ & 83.8 & 74.6 \\
\hline Initial $\mathrm{VA} \leq 0.25$ & $0.753(0.671-0.833)$ & $<0.001$ & 79.5 & 69.0 \\
\hline
\end{tabular}

AUC = Area under the curve (ROC); 95\% confidence intervals are given in parentheses

ables which were tested as potential prognostic factors for final VA in 1 year. An initial VA $\leq 0.1$ was significantly associated with major type of occlusion: $44(97.8 \%)$ versus 1 (2.2\%) in macular BRVO. A significantly higher incidence of ischemia was observed in major BRVO, with 52 $(92.6 \%)$ versus 4 (7.1\%) in macular BRVO. Age was found to be the most significant factor for most of the following examined variables: type of occlusion, grid laser photocoagulation, retinal ischemia and initial VA. For this reason, age was used in all model predictions of the final VA.

Table 2 summarizes univariate logistic regression models in terms of odds ratio and corresponding confidence intervals. The results indicate that only age and initial VA have a statistically significant association with the prognostic factors for final VA $\leq 0.1$. The other investigated factors did not reach a statistically significant odds ratio. Age was highly significantly associated both quantitatively in years and as a code around point 70 years. We performed the ROC analysis (table 3 ) to assess the diagnostic potential of investigated factors in relation to final VA. The ROC analysis confirmed the significant discrimination potential for final VA score in case of age $>70$ years and for categories of initial VA. All the other potential prognostic factors did not reach recognizable area-under-the-curve values, sensitivity and/or specificity. Regarding initial VA as a potential predictor, the ROC analysis proved the diagnostic power of category of initial VA $\leq 0.16$ as the most significant (it reached peak values of area under the curve, a sensitivity of $83.8 \%$ and a specificity of $74.6 \%$ ).

The multivariate logistic regression model for risk endpoint VA in 1 year $\leq 0.1$ showed that only age $>70$ years and initial VA $\leq 0.16$ were significant and mutu- ally independent terms of the predictive formula. The stepwise calculation procedure started with all examined variables, but none of the other variables reached significant multivariate adjusted odds ratios.

We can conclude that an initial VA $\leq 0.16$ was confirmed as a significant predictor of final VA $\leq 0.10$ in both univariate and multivariate models (not shown) and in the ROC analysis as well.

The other factors which were not included in the multivariate model (sex, occlusion type major/macular, grid photocoagulation yes/no and ischemia yes/no) did not reach a significant age-adjusted odds ratio.

As the initial VA significantly influenced the final visual result, we divided our BRVO patients according to initial VA into 3 subgroups: A $\geq 0.4$; B $0.3-0.16$, and C $\leq 0.1$. Median values and 5 th -95 th percentile range of $\mathrm{VA}$ in 1 year were as follows: $\mathrm{A}=0.66(0.10-1.00), \mathrm{B}=0.40$ (0.03-0.66), $\mathrm{C}=0.10(0.016-0.50)$. The final VA score was statistically significantly different among groups and the groups were found to be mutually statistically significantly different ( $<<0.001$; fig. 2$)$.

\section{Discussion}

Generally, the visual prognosis for patients with BRVO is relatively good but exact prognosis remains difficult. The most frequent cause of visual impairment is chronic ME which develops from complex intracellular and extracellular changes in a hypoxic retina [19]. Even when the occluded region does not directly involve the macula, other extracellular factors (such as vascular endothelial growth factor or interleukins) contribute to the 


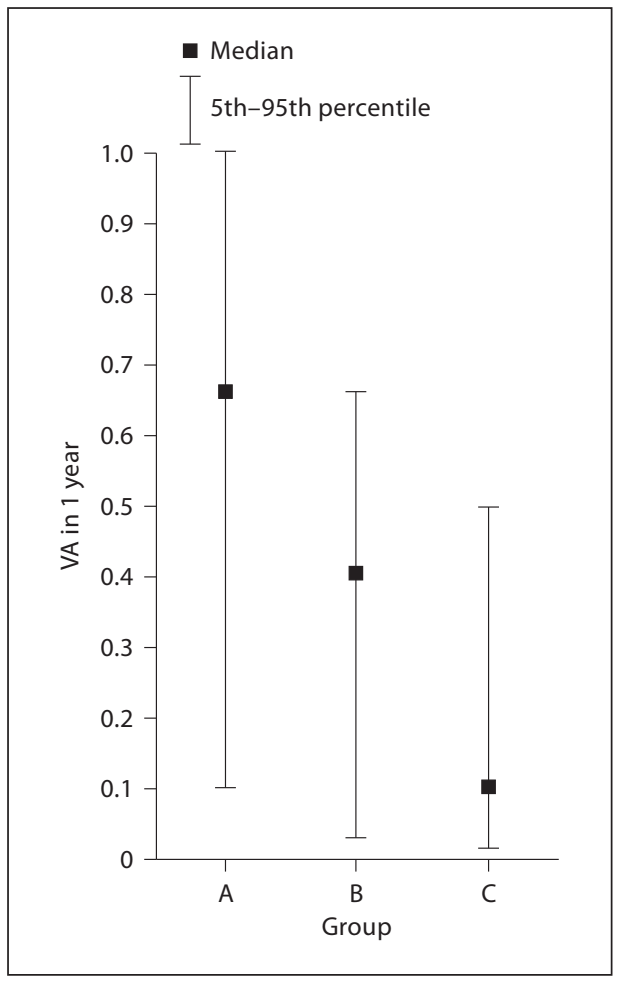

Fig. 2. The final VA 1 year after the inclusion into the study in groups $\mathrm{A}, \mathrm{B}$ and $\mathrm{C}$ dividing the patients according to the initial VA at the inclusion: $\mathrm{A} \geq 0.4 ; \mathrm{B} 0.3-0.16$; $\mathrm{C} \leq 0.1$. All groups are mutually statistically different with $\mathrm{p}<0.001$ (Mann-Whitney U test).

development of ME [20]. Some authors even claim that the course of the occlusion cannot be predicted $[2,21$, 22]. In our previous review of published studies [13], we showed that the percentage of a final VA $\leq 0.1$ was significantly higher in patients with an initial VA $\leq 0.1$ than in eyes with an initial VA $\geq 0.4$. Other authors have also speculated that pretreatment VA might influence the VA after laser photocoagulation in patients with $\mathrm{ME}$ due to BRVO [23]. Therefore, we performed the above retrospective analysis of our own BRVO patients with the aim of evaluating the role of initial VA as a prognostic factor for final VA.

The grid laser photocoagulation was indicated according to the recommendation of the BVO SG [21]. All patients also had subsidiary treatment with pentoxifylline, salicylic acid and ascorbic acid. Although the evidence for this subsidiary treatment is very weak, some authors have reported a possible benefit of rheological and antithrombotic therapy [24]. Since all eyes received this treatment, it did not influence the statistical evaluation.
Both univariate and multivariate analyses proved initial VA to be a significant predictor for the final VA. The ROC analysis confirmed an initial VA $\leq 0.16$ as the most significant predictor of risk VA outcome with the highest sensitivity and specificity. We can draw the important conclusion that pretreatment VA influences the final VA in patients with ME due to BRVO. We consider a BRVO prognostically unfavorable in terms of final visual result for those occlusions with an initial VA $\leq 0.16$. The ROC analysis also revealed that the prognostic value of initial VA is increased above a patient age $>70$ years. Our analyses also showed that sex, occlusion type, ischemic type of BRVO and grid laser treatment did not significantly contribute to the constructed models for predicting final VA.

The rate of poor final visual result $(\leq 0.1)$ varies widely in the literature from 12 to $46 \%[1-3,6,14-16,21,25]$. This fact points to methodological discrepancies in the way the results were evaluated in these studies. The rates of poor final VA in treated patients have been reported in some studies to be between 31 and $46 \%[14,22,25]$ and to exceed the rates in untreated groups of $19-23 \%[1,3,21]$. This might indicate that laser treatment leads to poorer visual results. The apparent paradox can be explained by our analyses. These studies differed considerably in the rate of eyes with poor initial VA, and, as we found, initial VA significantly influences the final visual result. If the study group comprises a higher percentage of eyes with poor initial VA, the poorer would be the prognosis. Esrick et al. [26] published data in which they used the same treatment schedule as the BVO SG [21], but the results differed significantly. Only $46.6 \%$ of the patients of Esrick et al. [26] gained 2 or more lines of vision compared to $65 \%$ if those of the BVO SG [21]. Esrick et al. [26] suggest that these differences may be due to a number of factors, including the poorer average prelaser VA in their patients. Glacet-Bernard et al. [11] evaluated prognostic factors in 48 cases of BRVO and concluded that older age and reduced baseline VA were correlated with poor visual prognosis. The results of our analysis are in agreement with the results of studies which evaluated predictive factors for visual improvement in patients with BRVO treated with bevacizumab $[27,28]$. Jaissle et al. [28] showed that younger patients ( $<60$ years) and initial VA $\leq 0.5$ $\log$ MAR (which correlates with $\geq 0.32$ in Snellen charts) are significantly associated with a good visual outcome. Therefore, the age and initial VA seem to be useful prognostic factors for the final VA not only in patients without any treatment or treated with grid laser photocoagulation, but also for cases treated with bevacizumab. 


\section{Conclusions}

This study shows that initial VA predicts final visual results in patients with BRVO. We consider a BRVO prognostically unfavorable for the final VA $(\leq 0.1)$ in patients with an initial VA of $\leq 0.16$. The results of this study and analysis of the published data suggest caution in the in- terpretation of different treatment evaluations in patients with BRVO. To compare the impact of two different treatment methods on VA, it is essential that the comparison is only among eyes with similar initial VA. We recommend dividing the patients with BRVO into 3 groups according to initial VA: $\geq 0.4,0.3-0.16$ and $\leq 0.1$.

\section{References}

1 Gutman FA, Zegarra H, Zakov ZN: The natural course of temporal retinal vein occlusion. Trans Am Acad Opthalmol Otolaryngol 1974;78:178-192.

-2 Magargal LE, Sanborn GE, Kimmel AS, Annesley WH: Temporal branch retinal vein obstruction: a review. Ophthalmic Surg 1986; 17:240-246.

3 Michels RG, Gass JDM: Natural course of temporal retinal branch occlusion. Trans Am Acad Ophthalmol Otolaryngol 1974;78: 166-177.

-4 Branch Vein Occlusion Study Group: Argon laser scatter photocoagulation for prevention of neovascularization and hemorrhage in branch vein occlusion. Arch Ophthalmol 1986;104:34-41.

5 Gutman FA: Macular edema in branch retinal vein occlusion: prognosis and management. Trans Am Acad Ophthalmol Otolaryngol 1977;83:488-493.

-6 Schilling JS, Jones CA: Retinal branch vein occlusion: a study of argon laser photocoagulation in the treatment of macular edema. Br J Ophthalmol 1984;68:196-198.

7 Chung EJ, Hong YT, Lee SC, Kwon OW, Koh HJ: Prognostic factors for visual outcome after intravitreal bevacizumab for macular edema due to branch retinal vein occlusion. Graefes Arch Clin Exp Ophthalmol 2008; 246:1241-1247.

8 Finkelstein D: Ischemic macular edema: recognition and favorable natural history in branch vein occlusion. Arch Ophthalmol 1992;110:1427-1434.

9 Scott IU, Van Veldhuisen PC, Oden NL, Ip MS, Blodi BA, Jumper JM, Figueroa M, SCORE Study Investigator Group: SCORE study report 1: baseline associations between central retinal thickness and visual acuity in patients with retinal vein occlusion. Ophthalmology 2009;116:504-512.
10 Ota M, Tsujikawa A, Kita M, Miyamoto K, Sakamoto A, Yamaike N, Kotera Y, Yoshimura N: Integrity of foveal photoreceptor layer in central retinal vein occlusion. Retina 2008;28:1502-1508

-11 Glacet-Bernard A, Coscas G, Chabanel A, Zourdani A, Lelong F, Samama MM: Prognostic factors for retinal vein occlusion: prospective study of 175 cases. Ophthalmology 1996; 103:551-560.

12 Central Vein Occlusion Study Group: Natural history and clinical management of central retinal vein occlusion. Arch Ophthalmol 1997;115:486-491.

13 Rehak J, Rehak M: Branch retinal vein occlusion: pathogenesis, visual prognosis, and treatment modalities. Curr Eye Res 2008;33: 111-131.

14 Wetzig PC: The treatment of acute branch vein occlusion by photocoagulation. Am J Ophthalmol 1979;87:65-73.

15 Jalkh AE, Avila PM, Zakka KA, Trempe CL, Schepens CS: Chronic macular edema in retinal branch vein occlusion: role of laser photocoagulation. Ann Ophthalmol 1984;16: 526-533.

16 Lang GE, Händel A: Ergebnisse der Laserkoagulation retinaler Venenastverschlüsse. Klin Monatsbl Augenheilkd 1993;203:180188.

17 Eng J: ROC analysis: web-based calculator for ROC curves. Baltimore, Johns Hopkins University (updated May 17, 2006). http:// www.jrocfit.org.

18 SPSS for Windows, release 12.0.1. Chicago, SPSS Inc, 2003.

19 Noma H, Funatsu H, Yamasaki M, Tsukamoto H, Mimura T, Sone T, Jian K, Sakamoto I, Nakano K, Yamashita H, Minamoto A, Mishima HK: Pathogenesis of macular edema with branch retinal vein occlusion and intraocular levels of vascular endothelial growth factor and interleukin-6. Am J Ophthalmol 2005;140:256-261.

-20 Rehak M, Hollborn M, Iandiev I, Pannicke T, Karl A, Wurm A, Kohen L, Reichenbach A, Wiedemann P, Bringmann A: Retinal gene expression and Müller cell responses after branch retinal vein occlusion in the rat. Invest Ophthalmol Vis Sci 2009;50:2359-2367.
21 Branch Vein Occlusion Study Group: Argon laser photocoagulation for macular edema in branch retinal vein occlusion. Am J Ophthalmol 1984;98:271-282.

22 Morse PH: Prospective rationale for and results of argon laser treatment of patients with branch retinal vein occlusion. Ann Ophthalmol 1985; 17:565-571.

23 Subramanian ML, Heier JS, Esrick E, Devaiah AK, Topping TM, Frederick AR, Morley MG: Preoperative visual acuity as a prognostic indicator for laser treatment of macular edema due to branch retinal vein occlusion. Ophthalmic Surg Lasers Imaging 2006;37:462-467.

24 Glacet-Bernard A, Coscas G, Chabanel A, Zourdani A, Lelong F, Samama MM: A randomized, double-masked study on the treatment of retinal vein occlusion with troxerutin. Am J Ophthalmol 1994;118:421-429.

25 Roseman RL, Olk RJ: Krypton red laser photocoagulation for branch retinal vein occlusion. Ophthalmology 1987;94:1120-1125.

26 Esrick E, Subramanian ML, Heier JS, Devaiah AK, Topping TM, Frederick AR, Morley MG: Multiple laser treatments for macular edema attributable to branch retinal vein occlusion. Am J Ophtalmol 2005; 139:653-657.

27 Kondo M, Kondo N, Ito Y, et al: Intravitreal injection of bevacizumab for macular edema secondary to branch retinal vein occlusion: results after 12 months and multiple regression analysis. Retina 2009;29:1242-1248.

28 Jaissle GB, Szurman P, Feltgen N, Spitzer B, Pielen A, Rehak M, Spital G, Heimann H, Meyer CH, Retinal Vein Occlusion Study Group: Predictive factors for functional improvement after intravitreal bevacizumab therapy for macular edema due to branch retinal vein occlusion. Graefes Arch Clin Exp Ophthalmol, submitted. 